\title{
Project RISARD - the story so far
}

\author{
Marcin P. Gawroński*, Krzysztof Goździewski, Krzysztof Katarzyński and Grzegorz \\ Rycyk \\ Centre for Astronomy, Faculty of Physics, Astronomy and Informatics, Nicolaus Copernicus \\ University, Grudziadzka 5, 87-100 Toruń, Poland \\ E-mail: motylek@astro.umk.p.*
}

In 2011 we have started new astrometric survey of active, young M dwarfs with the EVN at $6 \mathrm{~cm}$. The main goal of this project is to detect an exoplanet by direct, precise measurements of a M dwarf position and possible changes to this position caused by the planet. We summarize the current status of our survey and discuss the influence of the flux variability on the astrometry precision in the case of VLBI observations. We also present our estimation of the possible radio emission at $\mathrm{GHz}$ frequencies from young, massive exoplanets and show that it could be possible to obtain radio images of the gas giants/brown dwarfs using current global VLBI arrays.

12th European VLBI Network Symposium and Users Meeting,

7-10 October 2014

Cagliari, Italy

${ }^{*}$ Speaker. 


\section{Introduction}

RISARD (Radio Interferometric Survey of Active Red Dwarfs) is an astrometric project with the EVN at C-band started in 2011. This project is dedicated for observations of very young $(\lesssim 1 \mathrm{Gyr})$, low-mass stars placed within the distance range $10 \mathrm{pc}<d<15 \mathrm{pc}$. The main goal of this project is to detect an planet by direct, precise measurements of a $\mathrm{M}$ dwarf position and possible changes to this position caused by the planet. Selection of young stars increases the probability of planetary system occurrence rate because of increased mettalicity [16]. We target a region in physical and parameter space that cannot currently be probed by the radial velocity and/or transit method due to chromospheric activity of young M dwarfs. Moreover, the astrometric method may detect the stellar wobble around the barycenter independently of the orbital inclination. The first results show that planets within the Jupiter-Saturn mass range will be detectable [7], and a favorable sampling of the relative orbit would make it possible to determine the Keplerian elements already from 10 observational epochs (note that in this example, we assume the astrometric parameters pre-determined). We stress that our survey complements RIPL survey with the help of the VLBA [4]. RIPL is a program conducted at $4 \mathrm{~cm}$ wavelength, initiated in 2007 that is focused on 29 active $\mathrm{M}$ dwarfs within the distance $10 \mathrm{pc}$ from Sun.

Our astrometric survey consists of three stages and currently we are in the middle of third part of observations. We plan to finish observations in 2015. During the first stage we observed a new sample of 17 the most luminous X-ray M dwarfs. The selection was based on the empirical GüdelBenz relation between the X-ray and radio luminosity [3]. Next we performed regular astrometric survey of 12 detected stars during the initial part of the project (2012-2013, 3 epochs per target). In the final and third stage of RISARD we observe six M dwarfs which were detected in every epoch of realised so far observations (2013-2015, 5-6 epochs per target). Additionally, collected by us unique database of radio observations allows for study of radio activity of targeted during the RISARD M dwarfs.

\section{Impact of the flux variability on the VLBI astrometry precision}

We found that the high quality EVN data and the recently improved sensitivity of this network make it possible to study flux changes on short time-scales, even for sources with fluxes lower than $1 \mathrm{mJy}$. Thus, we are able to track the flux variability of the observed and detected $\mathrm{M}$ dwarfs from time-scales of $\sim 20 \mathrm{sec}$ in the case of $\sim 1$ mJy emission to time-scales of $\sim 3 \mathrm{~min}$ for stars with the fluxes at $\sim 200 \mu \mathrm{Jy}$ level. Here we present the complex changes in the radio flux of EQ Peg A observed by EVN at C-band on 2010 March 20 (Fig. 1).

In order to demonstrate the impact of radio variability for the astrometry precision we divided the observations collected for EQ Peg A into two sub-samples of equal duration and estimated separately the positions (see values in Tab. 1). The position error estimated with the use of the first sample is $20-30 \%$ lower than the error derived from the second set of data. Moreover, the error obtained for the first part is similar to the error derived for the whole set of the EQ Peg A observations. This is related to the fact that the average flux level in the first sub-sample was significantly higher than in the second sub-sample and hence connected with the different signalto-noise ratio in both parts of the data (Fig. 1). The lower signal-to-noise ratio in the second sub- 


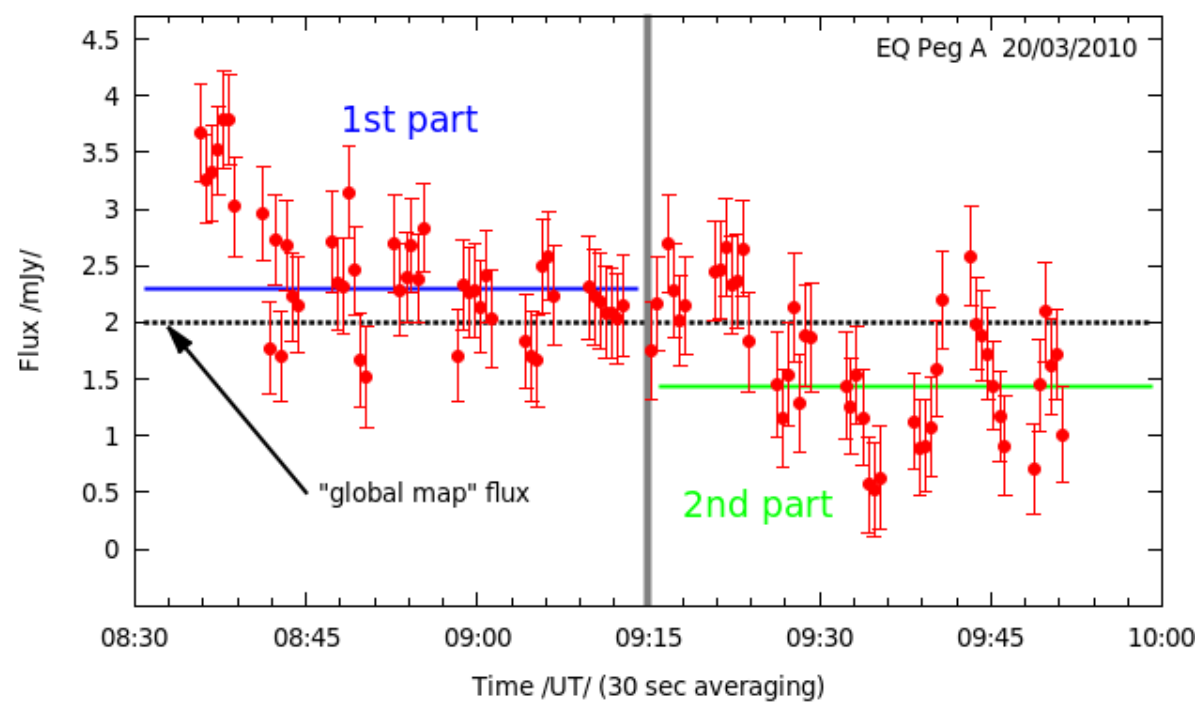

Figure 1: Changes in the total flux density of EQPeg A at $5 \mathrm{GHz}$ during the time of EVN observations on 2010 March 20. Each tick corresponds to $30 \mathrm{sec}$ bonds of data. Dashed black lines represent the radio flux measured from the map created with the use of whole dataset, blue and green line represents fluxes estimated from maps based on two equal sub-samples of data (blue - the first sub-sample, green - the second sub-sample). The gray line shows the border between two sub-samples.

sample affected the astrometry precision and decreased it. But in the case of the whole dataset and the first sub-samples the position errors are comparable. For presented here EQ Peg A observations the increase of integration time did not reflected in better astrometry precision. In a few hours long observations such changes in the radio flux may happen also a few times (even with dramatic flares present with the fluxes $\sim 2 \mathrm{mJy}$ ). Therefore, to increase precision of the astrometry is better to chose only the periods with relatively high radio flux levels if it is possible. Also an inspection of interferometric data in terms of variable signal may lead to improvement in the detection rate of $\mathrm{M}$ dwarfs. If it is assumed that during radio observations of an active $\mathrm{M}$ dwarf the star experienced high level of chromospheric activity, the rapid changes of the radio flux or even flares could be present in the data. If the duration of the activity or outburst is short in comparison to the integration time and the quiescent radio emission fall below the detection limit based on the whole dataset the the radio flux averaged during the mapping process could fall below the $3 \sigma$ limit. Thus, an object which may be detectable in a subset of the data would disappear when the whole dataset is used for mapping. Fortunately, ongoing sensitivity upgrades of VLBI networks should allow for detections on shorter time scales and hence reduce the impact of variability on stellar astrometry. Better sensitivity will also reduce the smearing effect that arises from stellar motion if the target proper motion occurred during the time of observations is comparable to the restoring beam.

\section{High frequency radio emission from young, massive exoplanets}

The theoretical models of the $\mathrm{MHz}$ radio emission from massive exoplanets were already investigated by several authors (e.g. [15]). It was also demonstrated that in principle, it should be possible to detect such emission from at least few known exoplanets (e.g. $\tau$ Boo b, $\varepsilon$ Eri b, Gl 86 b). 


\begin{tabular}{cccccc}
\hline \hline $\begin{array}{c}\text { Epoch } \\
(\mathrm{JD}-2455000)\end{array}$ & $\begin{array}{c}\mathrm{RA} \\
(\mathrm{J} 2000)\end{array}$ & $\begin{array}{c}\Delta \mathrm{RA} \\
(\mathrm{mas})\end{array}$ & $\begin{array}{c}\text { Dec } \\
(\mathrm{J} 2000)\end{array}$ & $\begin{array}{c}\Delta \mathrm{Dec} \\
(\mathrm{mas})\end{array}$ & $\begin{array}{c}S_{5 \mathrm{GHz}} \\
\mu \mathrm{Jy}\end{array}$ \\
\hline 275.88455 & 233152.594757 & 0.09 & 195613.466049 & 0.06 & $2005 \pm 103$ \\
\hline \hline $275.87083^{1)}$ & 233152.594760 & 0.10 & 195613.466002 & 0.06 & $2299 \pm 97$ \\
\hline $\left.275.89826^{2}\right)$ & 233152.594742 & 0.12 & 195613.466135 & 0.08 & $1422 \pm 80$ \\
\hline \hline
\end{tabular}

Table 1: Estimated positions of EQ Peg A based on EVN observations at C-band on 20 March 2010 (project code EG046). Errors in position are fitting errors and do not include any systematic effects. ${ }^{1)} \&{ }^{2)}$ Positions based on maps created from two subsamples of equal duration (1) $8^{h} 36^{m}-9^{h} 13^{m}$ and 2) $9^{h} 15^{m}-9^{h} 52^{m}$, times are given in UT).

However, so far there is no single confirmed detection of a radio emission from exoplanets despite many observational projects (e.g. [1,8]). Very recently it was claimed that sources of radio emission were found towards four planetary systems [17], but still these detections require further investigation because of probable background contamination. The expected maximal frequencies of radio emission from discovered so far exoplanets are below the L-band which is used by the existing global VLBI arrays and hence these instruments cannot be used in such studies. However, the fundamental question is if we can expect any radio emission from exoplanets at the $\mathrm{GHz}$ frequencies?

The main motivation for our calculations was the estimation of the magnetic field strength made by [15]. It was demonstrated there that in young $\left(\lesssim 10^{8} \mathrm{yrs}\right)$ and massive (from a few to several Jupiter masses) objects the magnetic fields strength may even excess $1 \mathrm{kG}$. In case of radio emission from the exoplanet the maximum frequency is simply the cyclotron frequency. Therefore radio emission observed in the Jupiter system at $\mathrm{MHz}$ frequencies could be generated in young exoplanets at $\mathrm{GHz}$ frequencies. Moreover the radio emission at $\gtrsim 1 \mathrm{GHz}$ frequency was discovered in the case of a few brown dwarfs (e.g. [2,9]) what supports existence of strong magnetic fields in massive planets/brown dwarfs. The main physical process which is responsible for observed radio emission from massive gas giants assumes interaction between the stellar wind of the host star and the planet magnetosphere. Such process is responsible for the most of the observed Jupiter's hectomeric emission. In order to estimate the magnetic field strength we use the approach proposed by [15]. They calculated the dynamo magnetic field strength at the surface of a planet using simple scaling relation showed by [14]. We also used the evolutionary models of massive planets/brown dwarf proposed by $[5,6]$.

We present here one of our simulations. Calculations were performed under assumption of a hypothetical planetary system placed in the distance $d=20 \mathrm{pc}$ and the radius of exoplanet orbit was assumed $r=1 \mathrm{AU}$. Changes in expected maximal flux and maximal frequency with the evolution of a massive exoplanet are shown on Fig. 2. Very massive gas giants with $M_{p}=40 M_{J u p}$ are able to produce radio emission at frequencies higher that $1.6 \mathrm{GHz}$ during first $\simeq 1 \mathrm{Gyr}$ of their evolution but for $M_{p}=20 M_{J u p}$ emission that could be observed at L-band is radiated only when the system is younger than $0.1 \mathrm{Gyr}$. However the estimated fluxes for the test system are at level of $0.3-0.8 \mathrm{mJy}$ what should be easily detectable by EVN and VLBA in a short $\sim 2 \mathrm{hrs} \mathrm{integration} \mathrm{time.}$ 


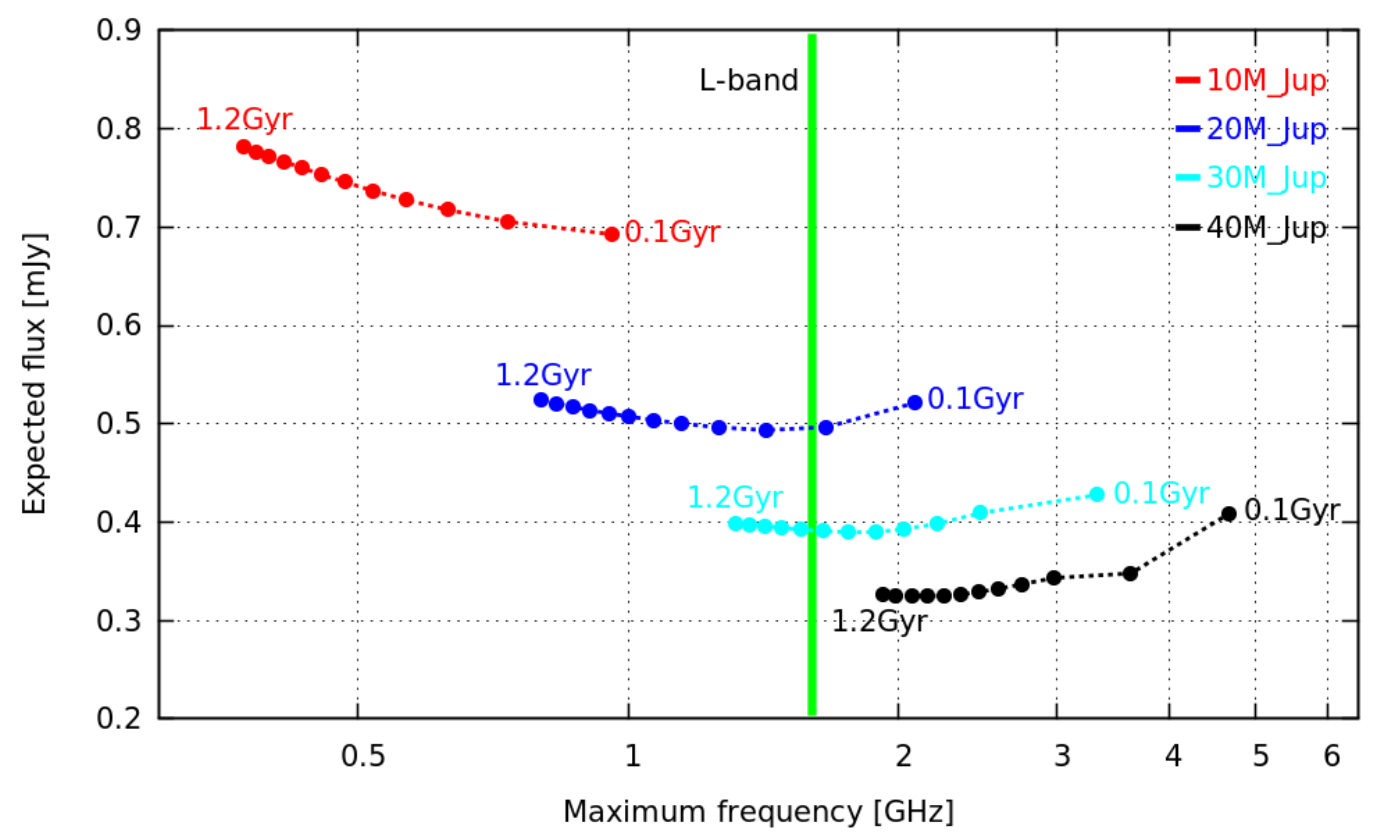

Figure 2: The maximal expected radio fluxes from a massive exoplanet located in a hypothetical planetary system placed in the distance $20 \mathrm{pc}$. It is assumed the the stellar wind particle density and velocity is five times greater than in case of the solar wind at the Jupiter orbit. The assumed size of the exoplanet orbit is 1 AU. Colours represent different exoplanet masses (red - 10M Jup , blue - 20M Jup , cyan - 30M Jup and black - $40 M_{\text {Jup }}$ ) and bold points show different system ages with the time steps 0.1 Gyr. The green line shows the L-band $(1.6 \mathrm{GHz})$ as a lower frequency limit for the EVN observations.

The global VLBI systems may be a perfect tool for searches of massive Jovian planets around the A-type main-sequence stars which are young objects $(\lesssim 1 \mathrm{Gyr})$ due to their fast evolution. Atype main-sequence stars have masses in the range of $1.4-2.1 M_{\odot}$. Because of the fundamental theoretical scaling relation between the stellar host mass and the protoplanetary disc mass (e.g. [11]) and the empirical relation between the giant planet frequency and the stellar host mass [10], selection of A-type stars increases the probability of the massive gas giants occurrence. Moreover the A-type main-sequence stars are usually fast rotators what reflects in almost featureless optical spectrum. This property of the A-type stars optical spectrum posses a big problem in case of standard radial velocity planetary surveys and these objects are frequently omitted from these projects. Currently our knowledge about planetary systems properties is limited only to direct imaging of exoplanets around A-type main-sequence stars and the use of the VLBI arrays could lead to important improvement in this field.

\section{Acknowledgement}

We are grateful to Polish National Science Centre for their support of project RISARD (grant no. 2011/01/D/ST9/00735). The EVN is a joint facility of European, Chinese, South African, and other radio astronomy institutes funded by their national research councils. 


\section{References}

[1] T.S. Bastian, G.A. Dulk and Y. Leblanc, A search for radio emission from extrasolar planets, ApJ, 545 (2000) 1058

[2] E. Berger, S. Ball, K.M. Becker, M. Clarke, D.A. Frail, T.A. Fukuda, I.M. Hoffman, R. Mellon, E. Momjian, N.W. Murphy, S.H. Teng, T. Woodruff, B.A. Zauderer, R.T. Zavala, Discovery of radio emission from the brown dwarf LP944-20, Nature, 410 (2001) 338

[3] E. Berger, G. Basri, T.A. Fleming, et al., Simultaneous Multi-Wavelength Observations of Magnetic Activity in Ultracool Dwarfs. III. X-ray, Radio, and Ho Activity Trends in M and L dwarfs, ApJ 709 (2010) 332

[4] G.C. Bower, A. Bolatto, E.B. Ford, P. Kalas, Radio Interferometric Planet Search. I. First Constraints On Planetary Companions For Nearby, Low-Mass Stars From Radio Astrometry, ApJ 701 (2009), 1922

[5] A. Burrows, W. Hubbard, D. Saumon and J.I. Lunine, An expanded set of brown dwarf and very low mass star models, ApJ, 406 (1993) 158

[6] A. Burrows, M. Marley, W.B. Hubbard, J.I. Lunine, T. Guillot, D. Saumon, R. Freedman, D. Sudarsky and C. Sharp, A nongray theory of extrasolar giant planets and brown dwarfs, ApJ, 491 (1997) 856

[7] M.P Gawro nski, K. Goździewski, K. Katarzynski, Project RISARD in proceedings of the 11th EVN Symposium, arXiv:1309:463

[8] S.J. George and I.R Stevens, Giant Metrewave Radio Telescope low-frequency observations of extrasolar planetary systems, MNRAS, 382 (2007) 455

[9] G. Hallinan, S. Bourke, C. Lane, A. Antonova, R.T. Zavala, W.F. Brisken, R.P. Boyle, F.J. Vrba, J.G. Doyle, A. Golden, Periodic bursts of coherent radio emission from an ultracool dwarf, ApJ, 663 (2007) 25

[10] J.A. Johnson, R.P. Butler, G.W. Marcy, D.A. Fischer, S.S. Vogt, J.T. Wright and K.M.G Peek, A new planet around an M Dwarf: Revealing a correlation between exoplanets and stellar mass, ApJ, 670 (2007) 833

[11] K. Kornet, S. Wolf and M. Różyczka, Formation of giant planets around stars with various masses, $A \& A, 458$ (2006) 661

[12] C. Marois, B. Macintosh, T. Barman, B. Zuckerman, I. Song, J. Patience, D. Lafreni Âĺre and R. Doyon, Direct imaging of multiple planets orbiting the star HR 8799, Science, 322 (2008) 1348

[13] J.D. Nichols, Magnetosphere-ionosphere coupling at Jupiter-like exoplanets with internal plasma sources: implications for detectability of auroral radio emissions, MNRAS 414 (2011) 2125

[14] A. Reiners, G. Basri and U.R. Christensen, Surprisingly weak magnetism on young accreting brown dwarfs, ApJ, 697 (2009) 373

[15] A. Reiners and U.R. Christensen, A magnetic field evolution scenario for brown dwarfs and giant planets, $A \& A, \mathbf{5 2 2}$ (2010) 13

[16] N.C. Santos, G. Isreaelian, M. Mayor, Spectroscopic [Fe/H] for 98 extra-solar planet-host stars. Exploring the probability of planet formation, A\&A 415 (2004) 1153

[17] S.K. Sirothia, A. Lecavelier des Etangs, Gopal-Krishna, N.G. Kantharia aand C.H. Ishwar-Chandra, $A$ search for radio emission from extrasolar planets, A\&A, 562 (2014) 108 